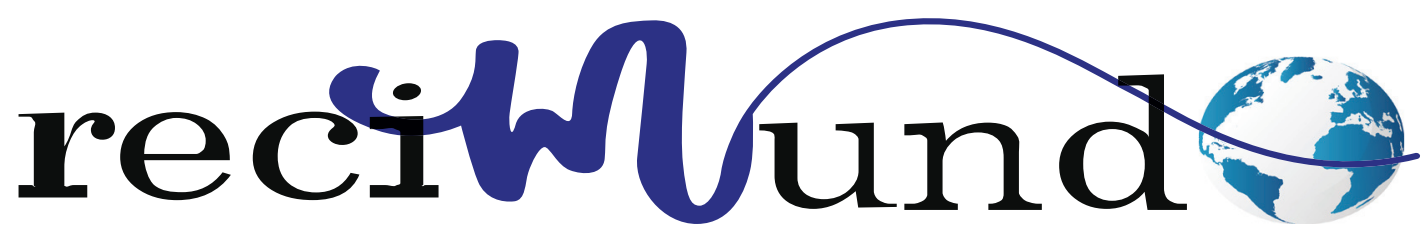

Revista Científica Mundo de la Investigación y el Conocimiento

DOI: 10.26820/recimundo/5.(Suple1).oct.2021.111-117

URL: https://recimundo.com/index.php/es/article/view/1339

EDITORIAL: Saberes del Conocimiento; Universidad Yachay Tech

REVISTA: RECIMUNDO

ISSN: 2588-073X

TIPO DE INVESTIGACIÓN: Artículo de revisión

CóDIGo UNESCO: 32 Ciencias Médicas

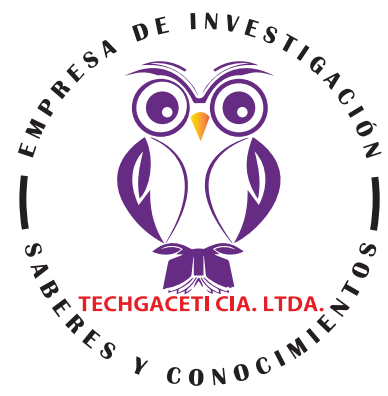

PAGINAS: $111-117$

\title{
Beneficios de la oxigenoterapia en pacientes con COVID-19
}

Benefits of oxygen therapy in patients with COVID-19

Benefícios da oxigenoterapia em pacientes com COVID-19

Kerlin Pamela Medina Ruela1; Damaris Alexandra Astudillo Junco²; Marjorie Jomira Coronado Macías ${ }^{3}$

RECIBIDO: 02/09/2021 ACEPTADO: 20/09/2021 PUBLICADO: 30/10/2021

1. Técnico Superior en Enfermería; Investigadora Independiente; Universidad Metropolitana de Ecuador; Guayaquil, Ecuador; kerlin.medina@est.umet.edu.ec; iD https://orcid.org/0000-0002-0592-5761

2. Técnico Superior en Enfermería; Investigadora Independiente; Universidad Metropolitana de Ecuador; Guayaquil, Ecuador; damaris.astudillo@est.umet.edu.ec; iD https://orcid.org/0000-0002-9463-1940

3. Técnico Superior en Enfermería; Investigadora Independiente; Universidad Metropolitana de Ecuador; Guayaquil, Ecuador; marjorie.coronado@est.umet.edu.ec; (D) https://orcid.org/0000-0002-1550-0820

CORRESPONDENCIA

Kerlin Pamela Medina Ruela

kerlin.medina@est.umet.edu.ec

Guayaquil, Ecuador

() RECIMUNDO; Editorial Saberes del Conocimiento, 2021 


\section{RESUMEN}

La finalidad de la oxigenoterapia es aumentar el aporte de oxígeno a los tejidos utilizando al máximo la capacidad de transporte de la sangre arterial. Muchas enfermedades, en particular las enfermedades pulmonares como el Covid- 19, reducen la cantidad de oxígeno en el torrente sanguíneo. En tales casos, la administración de oxígeno adicional puede resultar eficaz. Objetivo: Identificar los beneficios de la oxigenoterapia en los pacientes con Covid- 19. Material y método: se realizó una investigación de tipo documental, cualitativo con alcance descriptivo analítico, ya que se realizó la revisión de bibliografías de bases de datos empleando literatura nacional e internacional, en formato electrónico. Se emplearon un total de 10 referencias bibliográficas. Conclusión: La oxigenoterapia es un procedimiento dirigido a la prevención y el tratamiento de la hipoxemia, ya que aumenta el contenido de oxígeno en sangre arterial y permite un trabajo respiratorio eficiente garantizando niveles adecuados de saturación de oxígeno en estadios menos críticos de la enfermedad.

Palabras clave: Oxigenoterapia, Beneficios, Pacientes, Covid-19.

\section{ABSTRACT}

The purpose of oxygen therapy is to increase the supply of oxygen to the tissues by making the most of the transport capacity of arterial blood. Many diseases, particularly lung diseases like Covid-19, reduce the amount of oxygen in the bloodstream. In such cases, the administration of additional oxygen can be effective. Objective: To identify the benefits of oxygen therapy in patients with Covid-19. Material and method: a documentary, qualitative research with a descriptive analytical scope was carried out, since the bibliographies of databases were reviewed using national and international literature, in electronic format. A total of 10 bibliographic references were used. Conclusion: Oxygen therapy is a procedure aimed at the prevention and treatment of hypoxemia, since it increases the oxygen content in arterial blood and allows efficient work of breathing, guaranteeing adequate levels of oxygen saturation in less critical stages of the disease.

Keywords: Oxygen Therapy, Benefits, Patients, Covid-19

\section{RESUMO}

O objetivo da oxigenoterapia é aumentar o suprimento de oxigênio aos tecidos, aproveitando ao máximo a capacidade de transporte do sangue arterial. Muitas doenças, principalmente doenças pulmonares como Covid-19, reduzem a quantidade de oxigênio na corrente sanguínea. Nesses casos, a administração de oxigênio adicional pode ser eficaz. Objetivo: Identificar os benefícios da oxigenoterapia em pacientes com Covid-19. Material e método: foi realizada uma pesquisa documental, qualitativa, de abrangência analítica descritiva, uma vez que as bibliografias das bases de dados foram revisadas com recurso à literatura nacional e internacional, em formato eletrônico. Foram utilizadas 10 referências bibliográficas. Conclusão: A oxigenoterapia é um procedimento que visa a prevenção e tratamento da hipoxemia, pois aumenta o teor de oxigênio no sangue arterial e permite um trabalho respiratório eficiente, garantindo níveis adequados de saturação de oxigênio nas fases menos críticas da doença.

Palavras-chave: Terapia de oxigênio, Benefícios, Pacientes, Covid-19 


\section{Introducción}

COVID-19 es la enfermedad causada por el nuevo coronavirus conocido como SARSCoV-2. La Organización Mundial de la Salud (OMS) tuvo noticia por primera vez de la existencia de este nuevo virus el 31 de diciembre de 2019, al ser informada de un grupo de casos de «neumonía vírica» que se habían declarado en Wuhan (República Popular China). (1)

La oxigenoterapia se define como el aporte artificial de oxígeno (O2) en el aire inspirado; su objetivo principal es la oxigenación tisular, que se consigue cuando la presión parcial de $\mathrm{O} 2$ (pO2) en la sangre arterial supera los $60 \mathrm{mmHg}$., lo que se corresponde, aproximadamente, con una saturación de hemoglobina del 90\%. Hoy por hoy, la oxigenoterapia es la herramienta terapéutica fundamental en el tratamiento de los pacientes con insuficiencia respiratoria, tanto aguda como crónica. (2)

El oxígeno debe ser considerado un fármaco porque posee indicaciones precisas, debe ser utilizado en dosis y tiempo adecuados, posee efectos adversos y requiere criterios clínicos y de laboratorio para su evaluación. En el presente artículo se tiene como objetivo conocer los beneficios de la oxigenoterapia en pacientes con Covid, con el fin de desarrollar los factores que llevan a esta oxigenoterapia ser beneficiosa en esta patología. (2)

\section{Materiales y metodos}

El presente trabajo es de tipo documental, cualitativo con alcance descriptivo analíti$\mathrm{co}$, ya que se realizó la revisión de bibliografías de bases de datos como: Pagina de la Organización Mundial de la Salud, revistas científicas en las ciencias de la salud, NPunto anexada a LATINDEX, Scielo, Revista Cubana de Medicina Intensiva y Emergencias, tesis, Manual MSD y libros electrónicos, indagando trabajos publica- dos en relación a los beneficios de la oxigenoterapia en pacientes con covid-19, lo que permite el beneficio de estas herramientas digitales que permiten acceder a la información pertinente

\section{Desarrollo}

El COVID-19 fue declarado por la Organización Mundial de la Salud (OMS) el 11 de marzo del 2020 por emergencia con el máximo nivel de alerta preocupación internacional, siendo elevado como pandemia. Ese mismo día en Ecuador se emite la declaración del Estado de Emergencia Sanitaria en el Sistema Nacional de Salud, y el 16 de marzo se declara el estado de excepción, restricción de movilidad, suspensión de la jornada laboral y cierre de fronteras en el territorio ecuatoriano (3)

Desde luego muchas enfermedades, en particular las enfermedades pulmonares, reducen la cantidad de oxígeno en el torrente sanguíneo. En tales casos, la administración de oxígeno adicional puede resultar eficaz. Sin embargo, las pruebas científicas han demostrado que el oxígeno no es útil a menos que la concentración de oxígeno de una persona sea realmente baja. En realidad, respirar demasiado oxígeno puede dañar los pulmones al cabo de un tiempo. (4)

Alrededor del 19\% de los pacientes infectados por COVID19 van a presentar hipoxemia y necesidad de oxigenoterapia. La oxigenoterapia se utiliza como soporte, dando tiempo a que la etiología de la hipoxemia se revierta o se compense como en algunas patologías crónicas. El manejo de los pacientes con trastornos de la oxigenación, se debe llevar a cabo de manera ascendente, siempre y cuando las condiciones clínicas del paciente lo permitan. Iniciando con dispositivos de bajo flujo, alto flujo, cánula de alto flujo, ventilación mecánica no invasiva (VMNI), ventilación mecánica invasiva y por ultimo terapia de oxigenación extracorpórea (ECMO) (5) 
Puntualizando, la finalidad de la oxigenoterapia es aumentar el aporte de oxígeno a los tejidos utilizando al máximo la capacidad de transporte de la sangre arterial. Para ello, la cantidad de oxígeno en el gas inspirado, debe ser tal que su presión parcial en el alvéolo alcance niveles suficientes para saturar completamente la hemoglobina. Es indispensable que el aporte ventilatorio se complemente con una concentración normal de hemoglobina y una conservación del gasto cardíaco y del flujo sanguíneo hístico. La necesidad de la terapia con oxígeno debe estar siempre basada en un juicio clínico cuidadoso y fundamentado en la medición de los gases arteriales. El efecto directo es aumentar la presión del oxígeno alveolar, que atrae consigo una disminución del trabajo respiratorio y del trabajo del miocardio, necesaria para mantener una presión arterial de oxígeno definida. (2)

\section{Beneficios de la oxigenoterapia}

Se contempla un sin número de beneficios los que brinda el uso terapéutico del oxígeno para quienes padecen de patologías cardiopulmonares. Es importante resaltar que el beneficio principal es la mejoría en la calidad de vida, ya que el suministro de oxígeno permite generar más energía para realizar la contracción muscular y, por tanto, permite cubrir las demandas cinéticas en las actividades cotidianas, proporcionándole mayor independencia funcional al paciente. (6)

Dentro de los principales beneficios de la oxigenoterapia descritos en la literatura encontramos, entre otros, los siguientes:

1. Aumenta los niveles de PO2 arterial, lo que favorece una mejoría en la capacidad funcional residual.

2. Disminuye la disnea y la cianosis, pues a través del suministro de oxígeno se promueve una adecuada captación y transporte de oxígeno, lo que satisface las demandas metabólicas del organismo; a su vez, se mejora la perfusión capilar distal y se reduce la dificultad respiratoria, gracias al proceso de difusión.

3. Reduce la presión de las arterias pulmonares. El oxígeno es un elemento que en el organismo genera un efecto de vasodilatación, lo que reduce la presión que ejerce la sangre sobre las paredes vasculares pulmonares.

4. Mejora y mantiene la frecuencia cardiaca y respiratoria, pues el oxígeno reduce la presión vascular, lo que genera menor esfuerzo de la bomba cardiaca. De igual forma, al mejorar la relación ventilación perfusión, se mantiene la frecuencia cardiaca dentro de límites normales.

\section{Complicaciones del Covid- 19}

El síndrome respiratorio agudo severo por coronavirus 2 (SARS-CoV-2): Se ha definido la enfermedad por coronavirus 2019 (COVID-19) como una infección respiratoria aguda que potencialmente puede producir un síndrome respiratorio agudo severo producido por coronavirus2 (SARS-CoV-2), con un espectro clínico que va desde una enfermedad muy similar al refriado común hasta una neumonía grave e insuficiencia respiratoria aguda grave. (6) Criterios para el ingreso a UCl y uso de la oxigenoterapia

Se contemplan diversos criterios para el uso de la oxigenoterapia como en los casos confirmados graves que cumple con los criterios de ingresos en una unidad de cuidados intensivos, se ha observado en los pacientes que presentan un cuadro grave de la enfermedad una progresión de unos 10-15 días tras el inicio de síntomas hasta presentar SDRA, por lo que se requiere, al existir signos de gravedad, el ingreso precoz en una unidad de cuidados intensivos. (6) 
Entre los múltiples criterios para el uso de la oxigenoterapia se mencionan:

1. Disnea creciente. Taquipnea sostenida (frecuencia respiratoria $>25$ respiraciones / minuto).

2. Uso de músculos accesorios de la respiración, tiraje intercostal o subcostal.

3. Oximetría de pulso con saturación de oxígeno $\leq 92 \%$.

4. Estertores húmedos o secos en aumento. Cianosis.

5. Necesidad de ventilación artificial mecánica.

Partiendo de estos criterios la oxigenoterapia continua se comenzará a $5 \mathrm{~L} / \mathrm{min}$ con la meta de una SpO2 $292 \%-95 \%$ en mujeres embarazadas; en otros pacientes, el objetivo es una $\mathrm{SpO} 2 \geq 90 \%$. Además, se colocará monitor de parámetros fisiológicos. Oximetría de pulso permanente. Es imprescindible en control de signos vitales de inicio (frecuencia respiratoria, cardiaca, diuresis horaria, presión arterial, características del pulso, Ilene capilar, gradiente térmico, nivel de conciencia) y luego cada 1 hora en pacientes críticos, o según requiera la evolución médica. (6)

\section{Procedimiento de administración de oxí- geno}

Por ser el oxígeno un medicamento, debe ser este, administrado según cinco principios fundamentales que son: dosificado, continuado, controlado, atemperado y humidificado.

El estado del paciente, la causa y la severidad de la hipoxemia determinan fundamentalmente el método a usar para la administración de oxigenoterapia según el nivel de oxígeno en la sangre. El objetivo de este cubrir las necesidades de oxigeno del paciente, evitar sufrimiento tisular y disminuir el trabajo respiratorio y del miocardio. (2)

Relacionado con lo ya dicho la oxigenoterapia necesita ser Monitorizada puesto que el $\mathrm{O} 2$ es un medicamento y como tal, debe ser administrado con indicación y en dosis correcta, para evitar complicaciones. Su uso debe ser monitorizado. Esta monitorización se puede realizar mediante dos procedimientos: la gasometría arterial (método invasivo) y la oximetría de pulso (método no invasivo). Método no invasivo de monitorización, que permite detectar hipoxemia. Emite luz en dos longitudes de onda, la hemoglobina absorbe la luz y la transmite a un detector. El fotodetector mide la cantidad de luz que atraviesa los tejidos, y con la ayuda de un microprocesador se calcula el porcentaje de hemoglobina saturada (saturación de 02). (7)

\section{Control de la oxigenoterapia}

Se conoce que la $\mathrm{PaO} 2 / \mathrm{FiO} 2$ es un indicador muy preciso y eficaz de la función de la oxigenación por esto la estabilidad y el control del $\mathrm{FiO} 2$ son muy importante para pacientes en los que la enfermedad va progresando y que tienen un $\mathrm{PaO} 2 / \mathrm{FiO} 2$ por debajo de $300 \mathrm{mmHg}$. El tratamiento más indicado es la oxigenoterapia controlada.

Se recomienda la oxigenoterapia mediante una mascarilla nasal de flujo rápido (HFNC) para pacientes con las siguientes condiciones: $\mathrm{SpO} 2$ < 93 \%; PaO2/FiO2 < 300 mmHg ( $1 \mathrm{mmHg}=0,133 \mathrm{kPa}$ ); flujo respiratorio > 25 veces por min. en la cama; o evolución contrastada por rayo $\mathrm{X}$. Los pacientes han de usar una mascarilla quirúrgica durante el tratamiento HFNC. El flujo de aire de la oxigenoterapia HFNC debería empezar con un flujo lento y luego ir aumentando gradualmente hasta $40-60 \mathrm{~L} / \mathrm{min}$ cuando el $\mathrm{PaO} 2 / \mathrm{FiO} 2$ esté entre $200-300 \mathrm{mmHg}$ para que los pacientes no sufran opresión en el pecho y falta de respiración. Se debe ofrecer un flujo inicial de por lo menos $60 \mathrm{~L} / \mathrm{min}$ para pacientes con síndrome de dificultad respiratoria claro. (8)

Así pues, la intubación endotraqueal depende de la evolución de cada paciente, de

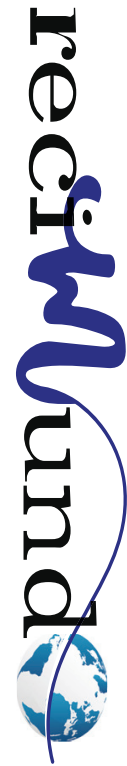


su condición sistémica, de las complicaciones de los pacientes, sobre todo de los que están estables, pero tienen una tasa de oxígeno baja (60 \%) HFNC. Los pacientes más mayores (> 60 años) con más complicaciones o PaO2/FiO2 de menos de $200 \mathrm{mmHg}$, deben trasladarse a la UCl. (8)

Desde luego es importante monitorizar las constantes, sobre todo la saturación de oxígeno por pulsioximetría (SpO2), para garantizar la seguridad durante las intervenciones en los casos que no se cuente con monitores ni telemetría. (9)

Destacando que la oxigenoterapia de alto flujo se presenta como una técnica sencilla, segura, con nulos efectos adversos. (10)

\section{Conclusiones}

El oxígeno es considerado un fármaco por tanto tiene indicaciones y efectos adversos con manifestaciones toxicas secundarias que se asocian a altas dosis y uso prolongado.

La oxigenoterapia es un procedimiento dirigido a la prevención y el tratamiento de la hipoxemia, ya que aumenta el contenido de oxígeno en sangre arterial y permite un trabajo respiratorio eficiente.

La oxigenoterapia garantiza niveles adecuados de saturación de oxígeno en estadios menos críticos de la enfermedad.

Desde el punto de vista hemodinámico, la distribución de oxigeno depende de la elación aporte/consumo, oxihemoglobina, presión parcial de oxígeno en las arterias, perfusión y la difusión de oxígeno.

Para elegir la forma adecuada de oxígeno se debe tener en cuenta los factores manifestados por el paciente, la patología y la respuesta ante la administración, comprobando la eficacia y la efectividad del tratamiento mediante la vigilancia de la satura- ción, el valor de oxígeno en la gasometría y demás signos y síntomas.

\section{Bibliografía}

Organización Mundial de la Salud. COVID 19. [Online].: OMS; [consultado 2021 Junio 19]; 2020. Available from: https://www.who.int/es/emergencies/ diseases/novel-coronavirus-2019/question-andanswers-hub/q-a-detail/coronavirus-disease-covid-19

Alonzo C, Peláez J, Sánchez J. La Oxigenoterapia En Pediatría Y Sus Complicaciones. NPunto. [Online] 2018; 1(5): p. Available from: https://www. npunto.es/revista/5/la- oxigenoterapia-en-pediatria-y-sus-complicaciones-5.

Bustamante Valencia C. Eficiencia De La Telemedicina En El Tratamiento Y Manejo Del Paciente Con Sospecha De Covid-19: Vinces - Ecuador. 2020 (Tesis Pregrado) Guayaquil: Repositorio Ug. Universidad de Guayaquil; [Online] 2020 Available from: http://repositorio.ug.edu.ec/bitstream/redug/49980/1/CD-181- BUSTAMANTE\%2OVALENCIA.pdf

Levine A, Stankiewicz J.; Tratamiento con oxígeno (oxigenoterapia). [Internet]. MSD Manual. 2020. Available from: https://www. msdmanuals.com/es-ec/hogar/trastornos- del-pulm\%C3\%B3n-y-las-v\%C3\%ADas-respiratorias/ rehabilitaci\%C3\%B3n-en-las- enfermedades-pulmonares-y-de-las-v\%C3\%ADas-respiratorias/tratamiento-con- ox\%C3\%ADgeno-oxigenoterapia

Ferrer L, Barrero J, Ballesteros O.; SOPORTE RESPIRATORIO EN PACIENTE CRÍTICO CON COVID-19. Colombia. 2020. [cited 2021] Available from: https://distribuna.com/wp-content/uploads/2021/02/ Libro-completo-Soporte- respiratoro-COVID-19. pdf

Pérez, A., Rivero, H., Pereda, R., Breto, A., Piloto, M., \& Oviedo, R. e7451 Guías de prácticas clínicas Protocolo para el tratamiento de la enfermedad por COVID-19 (SARS-CoV-2) en pacientes obstétricas ingresadas en cuidados intensivos. Revista Cubana de Medicina Intensiva y Emergencias. [Online] 2020;19(2): http://www.revmie.sld.cu/index.php/ mie/article/view/745/pdf

Pírez C, Peluffo G, Gustavo G, Menchaca A, Walter $P$, Karina $M$, et al. Oxigenoterapia. Scielo. 2020 Diciembre; 91(26-28.): p. http://www.scielo.edu. uy/scielo.php?script $=$ sci_arttext\&pid=S168812492020000700026.

LIANG T. Manual de Prevención y Tratamiento de COVID-19. [Online]. Zhejiang 2020. [cited 2021]. Available from: http://www.embajadachina.org.pe/ 
esp/sghd/P020200331264861016485.pdf

Guzmán Menéndez, GB; Morán Luna, L E. Evaluación de la condición funcional respiratoria y el impacto en la calidad de vida de los pacientes post covid-19, mediante la utilización de entornos virtuales. 2020 (TESIS PREGRADO) Guayaquil: Repositorio UCSG. Universidad Católica De Santiago De Guayaquil; [Online] 2020 Available from: http:// repositorio.ucsg.edu.ec/bitstream/3317/15401/1/ T-UCSG-PRE-MED-TERA- 229.pdf
Cobeña Intriago ME; Oxigenoterapia De Alto Flujo En Pacientes Postextubados En La Unidad De Cuidados Intensivos Pediátricos Del Hospital "Dr. Roberto Gilbert Elizalde" En El Periodo Julio - Diciembre Del 2016 (Tesis Posgrado) Guayaquil: Universidad Católica De Santiago De Guayaquil; [Online] 2017 Available from: http://repositorio.ucsg.edu.ec/bitstream/3317/9701/1/T-UCSG-PRE-EGM-ECIP-5.pdf

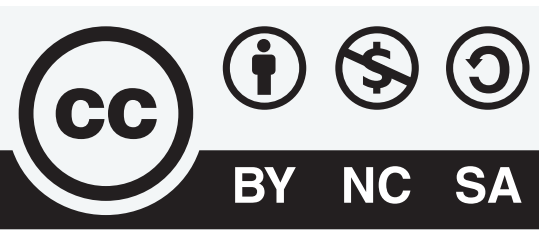

CREATIVE COMMONS RECONOCIMIENTO-NOCOMERCIAL-COMPARTIRIGUAL 4.0.

\section{CITAR ESTE ARTICULO:}

Medina Ruela, K. P., Astudillo Junco, D. A., \& Coronado Macías, M. J. (2021). Beneficios de la oxigenoterapia en pacientes con COVID-19. RECIMUNDO, 5(1 (Suple), 111-117. https://doi.org/10.26820/recimundo/5.(Suple1).oct.2021.111117 